\title{
In Brief Neuropsychological Assessment, Amnestic Mild Cognitive Impairment (MCI) Is associated with Cerebrospinal Fluid Biomarkers for Cognitive Decline in Contrast to the Prevailing NIA-AA MCI Criterion
}

Erik Hessen $^{\mathrm{a}, \mathrm{b}, *}$, Bjørn-Eivind Kirsebom ${ }^{\mathrm{c}, \mathrm{d}}$, Cecilia Magdalena Eriksson ${ }^{\mathrm{a}, \mathrm{b}, \mathrm{e}}$, Carl Fredrik Eliassen ${ }^{\mathrm{a}, \mathrm{b}}$, Arne Exner Nakling ${ }^{\mathrm{f}}$, Geir Bråthen ${ }^{\mathrm{g}, \mathrm{h}}$, Knut K. Waterloo ${ }^{\mathrm{c}, \mathrm{d}}$, Dag Aarsland ${ }^{\mathrm{a}, \mathrm{i}, \mathrm{j}}$ and Tormod Fladby ${ }^{\mathrm{a}, \mathrm{k}}$

${ }^{a}$ Department of Neurology, Akershus University Hospital, Lørenskog, Norway

${ }^{\mathrm{b}}$ Institute of Psychology, University of Oslo, Oslo, Norway

${ }^{\mathrm{c}}$ Department of Neurology, University Hospital of North Norway, Tromsø, Norway

${ }^{\mathrm{d}}$ Department of Psychology, Faculty of Health Sciences, UiT The Arctic University of Norway, Troms $\varnothing$

${ }^{\mathrm{e}}$ Department of Geriatric Psychiatry, Akershus University Hospital, Lørenskog, Norway

${ }^{\mathrm{f}}$ Betanien Hospital, Bergen, Norway

${ }^{\mathrm{g}}$ Department of Neuromedicine and Movement Science, Faculty of Medicine and Heath Sciences, Norwegian University of Science and Technology, Trondheim, Norway

${ }^{\mathrm{h}}$ Department of Neurology and Clinical Neurophysiology, University Hospital of Trondheim, Trondheim, Norway ${ }^{\mathrm{i}}$ Department of Old Age Psychiatry, Institute of Psychiatry, Psychology and Neuroscience, King's College London, London, UK

${ }^{\mathrm{j}}$ Center for Age-Related Diseases, Stavanger University Hospital, Stavanger, Norway

${ }^{\mathrm{k}}$ Institute of Clinical Medicine, Campus Ahus, University of Oslo, Oslo, Norway

\section{Abstract.}

Background: In the care of persons with cognitive problems, it is important to use a valid mild cognitive impairment (MCI) criterion that discriminates well between normal and pathological aging.

Objective: To find the brief neuropsychological screening criterion that best correlates with cerebrospinal fluid (CSF) biomarkers for cognitive decline and dementia in persons seeking help for cognitive problems.

Methods: 452 consecutively recruited patients (age 40-80 years) from memory-clinics in the Norwegian national multicentre longitudinal study Dementia Disease Initiation were included. CSF data as well as full data from brief neuropsychological screening were available for all patients.

\footnotetext{
${ }^{*}$ Correspondence to: Erik Hessen, Department of Neurology, Akershus University Hospital, PB 1000, 1478 Lørenskog, Norway. Tel.: +47 92097373; E-mail: erik.hessen@ @evropsykologi.no.
} 
Results: Amnestic MCI, including at least one memory test below T-score 40, outperformed the conventional US National Institute on Aging-Alzheimer's Association (NIA-AA) MCI criterion. Only amnestic MCI was significantly associated with biomarker pattern of NIA-AA stage 2 (low CSF A $\beta_{42}$ concentrations and elevated tau) in multivariate regression analysis.

Conclusions: The finding that amnestic MCI based on brief neuropsychological assessment is significantly associated with CSF biomarkers for cognitive decline and Alzheimer's disease is in accordance with longitudinal studies that find memory impairment; both in itself and especially in combination with other cognitive deficit to constitute a risk factor for subsequent cognitive decline and dementia. The prevalence of pathological biomarkers for Alzheimer's disease is common in the elderly and the clinical significance of present findings depend on longitudinal validation.

Keywords: Alzheimer's disease, amnestic MCI, brief neuropsychological assessment, cerebrospinal fluid biomarkers, mild cognitive impairment, NIA-AA MCI criterion, NIA-AA stage 2

\section{INTRODUCTION}

The prevailing definition of mild cognitive impairment (MCI) due to Alzheimer's disease (AD) [1] requires only one impaired neuropsychological test score, to any cognitive domain (memory, executive function, attention, language and visuospatial ability). According to this approach, impairment is typically defined by scores $1-1.5$ standard deviations (SD) or more below the mean for age- and educationmatched peers on culturally appropriate normative data.

However, this method has shown vulnerability to false-positive diagnostic errors [2-4]. With this approach, Edmonds et al. [4] observed a falsepositive MCI diagnosis in as many as $34.2 \%$ of Alzheimer's Disease Neuroimaging Initiative (ADNI) MCI cohort, and false-negative diagnostic errors in $7.1 \%$ of ADNI's cognitively normal control group. Due to these actuarial diagnostic problems, Loewenstein et al. [2], Jak et al. [5], and Bondi et al. [3] used an alternative classification. The Jak/Bondi criterion requires two or more tests in the same cognitive domain to be at least $\leq 1$ SD below the demographically normative mean scores $[3,5]$. Longitudinal findings show that, compared to the traditional criteria, this alternative criterion identifies more subjects that progress clinically and produces fewer subjects who return to normal neuropsychological function at follow-up [3]. One explanation for the poor diagnostic accuracy of the "one reduced test" approach is that cognitively healthy individuals may acquire poor test scores by chance when several cognitive tests are administered [6]. Of note, studies of patients with brain impairment of other etiology than neurodegenerative disease, do also suggest that the best balance between sensitivity and specificity is achieved with a mild cut off for impairment of 1 SD below the normative mean $[7,8]$.
While comprehensive neuropsychological assessment may be qualitatively better than a brief assessment with fewer tests [3], a disadvantage is that comprehensive testing is time consuming, which makes it less efficient and thus less available. For efficiency, many memory clinics therefore employ brief cognitive assessments and use the prevailing definition for MCI [1], despite evidence of diagnostic superiority of more comprehensive assessment with alternative impairment criteria [2-4].

In the study of patients presenting with neurodegenerative diseases, the most meaningful outcome is a valid prediction of longitudinal cognitive change. In a cross-sectional validation study of cognitive impairment criteria, a biomarker signature considered a risk factor for development of $\mathrm{AD}$ can be a surrogate for this goal. The US National Institute on Aging-Alzheimer's Association (NIA-AA) suggested in 2011 a "preclinical stage of AD", defined as patients without impaired cognition on standard assessments but positive biomarker evidence for $\mathrm{AD}$ [1]. According to this suggestion, stage 0 implies both amyloid and tau markers negative; stage 1 being lowered cerebrospinal fluid (CSF) amyloidbeta $(A \beta)_{42}$ concentrations but negative tau markers; stage 2, lowered CSF $A \beta_{42}$ concentrations and elevated tau concentrations; and stage 3 , biomarker pattern as in stage 2 with 'subtle cognitive decline' as evidenced by reduced neuropsychological test performance. In a recent longitudinal study $(n=122)$ following memory clinic patients on average 4 years without objective cognitive impairment, we found that biomarker-based classifications according to the NIA-AA 'preclinical AD' stage 2 was the best predictor of cognitive decline, dementia, and AD dementia [9]. A similar finding was reported in a five-year follow up [10] where a progression rate to probable mild dementia (CDR $\geq 0.5$ ) was $26 \%$ in participants classified as stage 2 at baseline, and $56 \%$ in those classified 
as stage 3. In a smaller 6-year longitudinal study of 81 memory clinic patients that only displayed subjective cognitive decline (SCD), findings suggested that pathological CSF $A \beta_{42}$ (similar to stage 1), predicted conversion to dementia [11]. This is in line with other studies [12,13]. With regard to prognosis of amnestic versus non-amnestic MCI, our previous longitudinal studies revealed that $\mathrm{MCI}$ with memory impairment, either as a single domain deficit or in the context of a multi-domain deficit, is a stronger predictor of cognitive decline and dementia than non-amnestic MCI $[15,16]$.

Based on the findings described above, we chose to validate different cognitive MCI criteria against the biomarker pattern of NIA-AA stage 2 (lowered CSF A $\beta_{42}$ concentrations and elevated CSF tau concentrations). We hypothesized that a MCI criterion, including mild amnestic deficit (Memory score 1 SD or more below normative mean), would show a stronger association with the biomarker pattern of NIA-AA stage 2, than both the conventional NIAAA MCI criterion and an MCI criterion similar to the criterion suggested by Jak et al. and Bondi et al. $[3,5]$.

\section{METHODS}

The study was approved by the regional medical research ethics committee. All participants gave their written informed consent before taking part. All further study conduct was in line with the guidelines provided by the Helsinki declaration of 1964; revised 2013 and the Norwegian Health and Research Act.

Patients were consecutively recruited from memory clinics and neurological centers that take part in the national multicenter longitudinal collaboration Dementia Disease Initiation (DDI), aiming at detection of early biological and cognitive markers for dementia. A detailed description of inclusion ad exclusion criteria is given in Fladby et al. [17]. Subjects in the present part of the study were recruited between January 2013 to November 2017. In summary, inclusion criteria were age $40-80$ years, recent appearance of cognitive concerns or symptoms and a native language of Norwegian, Swedish, or Danish. Exclusion criteria were brain trauma or disorder, including clinical stroke, dementia, severe psychiatric and/or somatic disease that may account for symptoms, intellectual disability or other development disorder.
The DDI-study employ the NIA/AA [1] and Jessen et al. [18] criteria for stage classification, and disease diagnosis. By December 2017, CSF data as well as full data from brief cognitive screening was available for 452 patients with cognitive concerns or symptoms.

\section{Clinical assessment}

A Case Report Form (CRF) was completed for all the patients, including standardized assessments of medical history from subject and informant, physical and neurological examinations, including the MiniMental State Examination (MMSE) [19], Geriatric Depression Scale (GDS), and brief neuropsychological screening. The brief neuropsychological assessment includes six cognitive functions. All the test scores were converted to T-scores based on available normative data.

1. Delayed verbal recall (Consortium to Establish a Registry for Alzheimer's Disease (CERAD) word list) [20, 21].

2. Delayed verbal recognition (CERAD word list) $[20,21]$.

3. Visuoperceptual ability (Visual Object Space Perception (VOSP) silhouettes) [22].

4. Psychomotor speed (Trail Making A, TMT-A) [23].

5. Cognitive flexibility/divided attention (Trail Making B, TMT-B) [23].

6. Phonetic word fluency (Controlled Oral Word Association Test, COWAT) [24].

We tested the three following neuropsychological algorithms against the NIA-AA stage 2:

(1) A stringent version of the NIA-AA MCI criterion [1], as employed in the DDI study: at least one test score in any cognitive domain similar to or below T-score 35 .

(2) A criterion similar to the suggestion of Jak et al. [5] and Bondi et al. [3]: at least two test scores in any cognitive domain below T-score 40.

(3) Amnestic MCI: at least one test score below T-score 40, including at least one memory test.

Additionally, these algorithms were tested independently against the criteria for abnormal $A \beta_{42}$, total tau (T-tau), and phosphorylated tau (P-tau) [25, 26]. 


\section{Biomarkers}

CSF samples were obtained by lumbar puncture using a standardized protocol. Lumbar puncture was performed before noon, and CSF was collected in polypropylene tubes (Thermo Nunc) and centrifuged within $4 \mathrm{~h}$ at $2000 \mathrm{~g}$ for $10 \mathrm{~min}$ at room temperature. The supernatant was transferred to new tubes and frozen at $-80^{\circ} \mathrm{C}$ prior to analysis. All CSF samples were analyzed at the Department of Interdisciplinary Laboratory Medicine and Medical Biochemistry at Akershus University Hospital, and samples from other sites were frozen before sending to this laboratory. We analyzed CSF concentrations of T-tau, P-tau 181 , and $A \beta_{42}$ by using ELISA (Innotest $\beta$ Amyloid (1-42), Innotest h-Tau Ag and Innotest Phospho-Tau (181P), Fujirebio, Ghent Belgium. The following cut-off values for CSF T-tau and P-tau abnormality were applied according to the laboratory recommendations (modified from Sjögren et al. 2001) [25]; t-tau $>300 \mathrm{pg} / \mathrm{ml}$ for age $<50$ years, $>450$ $\mathrm{pg} / \mathrm{ml}$ for age 50-69 years, and $>500 \mathrm{pg} / \mathrm{ml}$ for age $\geq 70$ years and $\mathrm{p}$-tau $\geq 80 \mathrm{pg} / \mathrm{ml}$. An optimal cut-off at CSF $A \beta_{1-42}<708$ for amyloid plaque pathology was determined following DDI PET $\left[{ }^{18} \mathrm{~F}\right]$-Flutemetamol uptake studies [26]. NIA-AA stage 2 classification have both pathological $A \beta_{42}$ and T-tau, showing evidence of amyloidosis and neurodegeneration.

\section{Statistics}

Descriptive statistics of the demographic, clinical, behavioral, and cognitive characteristics of the patient population was analyzed. Group comparisons were tested using Pearson's Chi-square for categorical variables and independent samples $t$-tests for continuous variables. Odds ratios for occurrence of NIA-AA stage 2, based on the three neuropsychological algorithms specified in the introduction as well as age and years of education was estimated with logistic regression analysis. Univariate analyses were first performed for variables reported in Table 3, and significant factors were included in the multivariate analysis. Results of the multivariate analysis are presented with odds ratios (OR) with $95 \%$ confidence intervals $(\mathrm{CI})$ and $p$-values. All tests were performed at a $5 \%$ significance level. Similar analyses were performed with the employed criteria for abnormal $\mathrm{A} \beta_{42}$, T-tau, and P-tau as dependent variables. The Statistical Package for Social Sciences (SPSS) version 25 was used.

\section{RESULTS}

Demographic, clinical, and cognitive scores for the patient population are provided in Table 1. Table 2 shows scores for patients fulfilling the biomarker pattern of NIA-AA stage 2 criterion $(n=53)$ or not $(n=398)$. The two groups are significantly different on most of the variables, but similar with regard to gender, years of education as well as on three of the six neuropsychological tests (COWAT, TMT-A, and VOSP silhouettes).

Univariate logistic regression analysis showed significant associations with the dependent variable (NIA-AA stage 2) for amnestic MCI $(n=144)$ $(\mathrm{OR}=5.7$, CI 3.1-10.6, $p=0.001)$, age at inclusion $(\mathrm{OR}=1.1$, CI 1.0-1.1, $p=0.001)$, for the MCI condition requiring two or more tests below $\mathrm{T}=40$ in any cognitive domain $(n=152)(\mathrm{OR}=3.9$, CI 2.1$7.0, p=0.001$ ) and for the condition requiring one test or more similar to or below $\mathrm{T}=35$ in any

Table 1

Demographic, clinical, and cognitive scores for the patient population

\begin{tabular}{ll}
\hline Variables & All patients $(n=452)$ \\
\hline Mean age (range) (SD) & $63.2(40-84)(9.3)$ \\
Female (\%) & 49.3 \\
Education, y (range) (SD) & $13.7(7-22)(3.3)$ \\
MMSE (range) (SD) & $28.5(20-30)(1.8)$ \\
$\mathrm{A} \beta_{42}$, ng/l (range) (SD) & $955.28(300-1880)(291.0)$ \\
$\mathrm{T}-$ tau, ng/l (range) (SD) & $376.71(75-1370)(226.8)$ \\
P-tau, ng/l (range) (SD) & $59.66(16-185)(28.1)$ \\
CERAD, recall, T-score (range) (SD) & $46.8(14-69)(14.4)$ \\
CERAD, recognition, T-score (range) (SD) & $46.7(10.0-57.6)(14.7)$ \\
COWAT, T-score (range) (SD) & $48.4(24-77)(9.9)$ \\
TMT-A, T-score (range) (SD) & $45.2(12-74)(10.5)$ \\
TMT-B, T-score (range) (SD) & $46.1(9-76)(11.3)$ \\
VOSP silhouettes, T-score (range) (SD) & $48.5(15-70)(11.2)$ \\
\hline
\end{tabular}


Table 2

Demographic, clinical, and cognitive scores for patients fulfilling NIA-AA stage 2 criterion or not

\begin{tabular}{lccr}
\hline Variables & $\begin{array}{c}\text { Fulfilling NIA-AA stage 2 } \\
(n=53)\end{array}$ & $\begin{array}{c}\text { Not fulfilling NIA-AA stage 2 } \\
(n=398)\end{array}$ & $p$ \\
\hline Mean age (range) (SD) & $67.8(50-81)(7.8)$ & $62.6(40-84)(9.3)$ & 0.001 \\
Male/female (n) & $197 / 200$ & $31 / 22$ & 0.45 \\
Education, y (range) (SD) & $13.9(7-20)(3.4)$ & $13.7(7-22)(3.3)$ & 0.78 \\
MMSE (range) (SD) & $26.9(21-30)(2.3)$ & $28.7(20-30)(1.6)$ & 0.001 \\
A $\beta_{42}$, ng/l (range) (SD) & $557.3(300-700)(95.1)$ & $1008.3(300-1880)(266.1)$ & 0.001 \\
T-tau, ng/l (range) (SD) & $783.0(443-1370)(237.4)$ & $322.6(75-1170)(160.4)$ & 0.001 \\
P-tau, ng/l (range) (SD) & $108.1(63-185)(31.4)$ & $53.2(16-185)(20.4)$ & 0.001 \\
CERAD, recall, T-score (range) (SD) & $34.5(14-69)(15.2)$ & $48.5(14-69)(13.5)$ & 0.001 \\
CERAD, recognition, T-score (range) (SD) & $26.5(10.0-58)(17.6)$ & $48.3(10-58)(13.6)$ & 0.001 \\
COWAT, T-score (range) (SD) & $49.0(26-69)(9.5)$ & $48.3(24-77)(10.0)$ & 0.62 \\
TMT-A, T-score (range) (SD) & $43.6(12-66)(10.4)$ & $45.4(14-74)(10.5)$ & 0.24 \\
TMT-B, T-score (range) (SD) & $41.7(9-70)(14.4)$ & $46.6(11-76)(10.8)$ & 0.02 \\
VOSP silhouettes, T-score (range) (SD) & $46.2(22-70)(11.0)$ & $48.8(15-70)(11.3)$ & 0.12 \\
\hline
\end{tabular}

Table 3

Univariate and multivariate regression analysis [dependent variable: NIA-AA stage $2=$ lowered cerebrospinal fluid (CSF) $\mathrm{A} \beta_{42}$ concentrations, and neurodegeneration (elevated CSF tau concentrations)]

\begin{tabular}{|c|c|c|c|c|}
\hline Variable & $\begin{array}{c}\text { Univariate } \\
\text { Analysis } \\
\text { OR }(95 \% \mathrm{CI})\end{array}$ & $p$ & $\begin{array}{c}\text { Multivariate } \\
\text { Analysis } \\
\text { OR }(95 \% \text { CI })\end{array}$ & $p$ \\
\hline $\begin{array}{l}\text { One test or more }<\mathrm{T}=40 \text {, including at least } \\
\text { one memory test }\end{array}$ & $5.7(3.1-10.6)$ & 0.001 & $4.4(1.9-10.4)$ & 0.001 \\
\hline Age at inclusion & $1.1(1.0-1.1)$ & 0.001 & $1.1(1.0-1.1)$ & 0.007 \\
\hline $\begin{array}{l}\text { Two or more tests }<\mathrm{T}=40 \text { in any cognitive } \\
\text { domain }\end{array}$ & $3.9(2.1-7.0)$ & 0.001 & $2.2(0.9-5.5)$ & 0.093 \\
\hline $\begin{array}{l}\text { One test or more }<\mathrm{T}=35 \text { in any cognitive } \\
\text { domain (NIA-AA MCI criterion) }\end{array}$ & $2.9(1.6-5.4)$ & 0.001 & $0.5(0.2-1.5)$ & 0.243 \\
\hline Years of education & $1.0(0.9-1.1)$ & 0.77 & & \\
\hline
\end{tabular}

cognitive domain (NIA-AA MCI criterion) $(n=204)$ $(\mathrm{OR}=2.9$, CI 1.6-5.4, $p=0.001)$ (Table 3). Only the amnestic variant of MCI $(\mathrm{OR}=4.4, \mathrm{CI} 1.9-10.4$, $p=0.001)$ and age at inclusion $(\mathrm{OR}=1.1, \mathrm{CI} 1.0-1.1$, $p=0.007)$ remained significant in the multivariate analysis. Similar results were revealed both in univariate and in multivariate analysis with abnormal levels of T-tau, P-tau, and $A \beta_{42}$ as dependent variables. In multivariate analysis the association was significant for all the variables with the amnestic variant of MCI, T-tau (OR =3.1, CI 1.7-5.9, $p=0.001)$, P-tau $(\mathrm{OR}=2.1$, CI 1.1-4.1, $p=0.023)$, and $\mathrm{A} \beta_{42}$ $(\mathrm{OR}=2.2$, CI 1.2-4.0, $p=0.01)$. Similar findings were evident for age at inclusion, T-tau $(\mathrm{OR}=1.0, \mathrm{CI} 1.0-$ $1.1, p=0.014), \mathrm{P}-\operatorname{tau}(\mathrm{OR}=1.1, \mathrm{CI} 1.0-1.1, p=0.001)$ as well as $\mathrm{A} \beta_{42}(\mathrm{OR}=1.1$, CI 1.0-1.1, $p=0.001)$. Additionally, abnormal level of $A \beta_{42}$ was in multivariate analysis significantly associated with the MCI condition requiring two or more tests below $\mathrm{T}=40$ in any cognitive domain $(\mathrm{OR}=2.3, \mathrm{CI} 1.2-4.4$, $p=0.017)$.

\section{DISCUSSION}

The main finding of this study is that amnestic MCI, including at least one memory test below T-score 40, outperformed both the conventional NIAAA MCI criterion and the MCI condition similar to the criterion suggested by Bondi et al. and Edmonds et al. [3, 4]. Amnestic MCI was the only MCI criterion that was significantly associated with the biomarker pattern of NIA-AA stage 2 in multivariate regression analysis. While the odds ratio for occurrence of NIAAA stage 2 was high (OR: 4.4) for patients classified according to amnestic MCI, it was associated with a low probability of NIA-AA stage 2 (OR: 0.5 ) for patients classified according to the conventional NIAAA MCI criterion. Age at inclusion was also slightly associated (OR: 1.1) with the biomarker pattern of NIA-AA stage 2.

Similar results were also evident when the same independent variables (age, years of education and abnormal levels of CSF T-tau, P-tau, and $\mathrm{A} \beta_{42}$ ) were 
tested independently in multivariate analysis against the employed MCI criteria. Of particular interest is that the conventional MCI criterion was not associated with any of the independent variables in the multivariate analysis.

The NIA-AA stage 2 has been found to increase the risk of subsequent cognitive decline in patients with no or only subjective cognitive decline at baseline $[9,10]$. The rather mild amnestic impairment criterion used in this study showed a stronger association with the biomarker-pattern characteristic of this stage, than the most commonly used NIA-AA MCI criterion [1] and a variant of the Jak/Bondi criterion [5, 3]. This suggests that the amnestic MCI criterion as applied here is precise and possibly predictive of later cognitive decline in cases where a brief neuropsychological protocol is available. The association of the amnestic MCI criterion with lowered CSF $A \beta_{42}$ concentrations and elevated CSF tau concentrations (as in NIA-AA stage 2), suggests that those who display mild memory impairment are most likely to also display hallmark AD biomarker pathology, according to the NIA-AA suggestion from 2011. However, it is necessary to bear in mind that the amyloid cascade hypothesis, implying lowered CSF $A \beta_{42}$ concentrations as the initial sequence of events in $\mathrm{AD}$, recently has been challenged. Studies have found low correlations between AD biomarkers across disease stages [27], a considerable proportion of subjects with MCI develop $\mathrm{AD}$ in the absence of pathological amyloid $[10,28]$, and many elderly have lower CSF A $\beta$ levels but normal cognitive function [29]. A recent Swedish study found that $46 \%$ of cognitively well-functioning 70 year-olds had at least one pathological AD biomarker [30]. While associations between MCI classifications and biomarkers are informative, the true test and validation of MCI criteria requires longitudinal follow up. With slightly different test batteries, our previous longitudinal studies showed that MCI with memory impairment, either as a single domain deficit or in the context of a multi-domain deficit, is a stronger predictor of subsequent cognitive decline and dementia than non-amnestic MCI $[15,16]$. This indicates that the present findings may have some interest beyond the association with pathological CSF biomarkers at baseline. In a two-year follow up study, Hessen et al. [15] found that 29\% percent of patients with pure amnestic MCI and 59\% percent of patients with amnestic, multi domain MCI converted to dementia as opposed to only $10 \%$ of patients with non-amnestic MCI. In the present study, both the NIA-AA MCI criterion [1] and a variant of the criterion suggested by Jak et al. and Bondi et al. [3, 5] do not require memory problems and thus, may contain many subjects with possibly more benign non-amnestic MCI.

The relatively poor association, in a multivariate context, between the condition similar to the criterion suggested by Jak et al. and Bondi et al. [3, 5] (in this study, at least two tests in any cognitive domain below T-score 40) and the biomarker pattern of NIA-AA stage 2, may be explained by the different preconditions in this study and the original works by Jak et al. [5] and Bondi et al. [3]. They employed somewhat more comprehensive neuropsychological assessment than the brief assessment in this work. Furthermore, their seemingly lenient MCI criterion (only $1 \mathrm{SD}$ below expected mean) is possibly not so lenient, as this criterion differ from other criteria with the requirement of 2 mildly impaired neuropsychological tests scores $(-1 \mathrm{SD})$ in the same cognitive domain. Based on this they achieved a robust MCI score with a higher percentage of stable MCI subjects (i.e., did not revert back to normal test scores at follow up) and a higher percentage of subjects that converted to dementia at follow-up, than patients classified according to more typical MCI criteria [1]. This kind of criterion has also shown distinct cortical atrophy characteristics, not captured by the traditional criteria [31].

Previous reports seem to suggest that a comprehensive neuropsychological assessment is superior to brief cognitive assessments. However, this is not universally supported. One study found that single neuropsychological tests can be superior to biomarkers as predictors of cognitive decline, in particular tests of learning and memory [32]. Another study that explored the best combination of multiple AD biomarkers and neuropsychological tests in predicting subsequent decline, found that CSF ttau/amyloid and MRI biomarkers, together with the combination of only two neuropsychological tests of memory and executive function were the best predictors [33]. Among several single-predictor models (biomarkers and neuropsychological test scores), both entorhinal cortex volume and TMT-B had similar predictive utility as multimarker predictors at determining those that would deteriorate clinically over 3 years [33]. Similar utility of neuropsychological testing as compared with neurobiological metrics have been reported in several other studies [34-36]. Taken together, these findings suggests that the prediction of subsequent decline in the context 
of MCI do not necessarily require a comprehensive neuropsychological evaluation, supporting further exploration of the utility of brief assessments in this endeavor.

The present study has some limitations. Our motivation for utilizing NIA-AA 'preclinical AD' stage 2 [1] is that this biological profile was the best predictor of cognitive decline, dementia, and $A D$ dementia in our 4-6 year follow up of SCD patients [9]. NIA-AA recently published an updated research framework based on a biological definition of $\mathrm{AD}$ to replace the guidelines from 2011 [37]. Imaging and/or biofluid biomarkers for $A \beta$ deposition (A), neurofibrillary pathology $(\mathrm{T})$, and neurodegeneration $(\mathrm{N})$ are categorized as separate biomarker signatures [AT (N)], the first two $(\mathrm{A}, \mathrm{T})$ as AD-specific whereas the third $(\mathrm{N})$ signifies neurodegeneration that also may be seen in other diseases. Thus, a possible problem with the NIA-AA 'preclinical AD' stage 2 is the inclusion of markers both specific for AD (lowered CSF $A \beta_{42}$ and elevated CSF P-tau) as well markers that also is associated with other etiologies than AD (elevated CSF T-tau) [37]. As the novel research framework allows for better differentiation between AD specific markers and markers associated with suspected non-Alzheimer pathologic change, this framework has a probable advantage over the older classification, and should be validated against cognitive signatures and criteria for cognitive impairment, and tested in future longitudinal studies of persons at risk for cognitive decline and dementia. Another limitation of the study is the cross-sectional design that precludes interpretation of how cognitive functioning develops during the course of the disorder.

In conclusion, we found that amnestic MCI based on brief neuropsychological testing and a MCI criterion customized to this test battery is significantly associated with CSF biomarkers for cognitive decline. In contrast, when we used the prevailing NIA-AA MCI criterion, non-significant associations were found. However, since the prevalence of pathological biomarkers for cognitive decline and $\mathrm{AD}$ is very common, the clinical significance of the present findings depends on longitudinal validation.

\section{DISCLOSURE STATEMENT}

Authors' disclosures available online (https:// www.j-alz.com/manuscript-disclosures/18-0964r1).

\section{REFERENCES}

[1] Albert MS, DeKosky ST, Dickson D, Dubois B, Feldman HH, Fox NC, Gamst A, Holtzman DM, Jagust WJ, Petersen RC, Snyder PJ, Carrillo MC, Thies B, Phelps CH (2011) The diagnosis of mild cognitive impairment due to Alzheimer's disease: Recommendations from the National Institute on Aging-Alzheimer's Association workgroups on diagnostic guidelines for Alzheimer's disease. Alzheimers Dement 7, 270-279.

[2] Loewenstein DA, Acevedo A, Small BJ, Agron J, Crocco E, Duara R (2009) Stability of different subtypes of mild cognitive impairment among the elderly over a 2- to 3-year follow-up period. Dement Geriatr Cogn Disord 27, 418423.

[3] Bondi MW, Edmonds EC, Jak AJ, Clark LR, DelanoWood L, McDonald CR, Nation DA, Libon DJ, Au R, Galasko D, Salmon DP (2014) Neuropsychological criteria for mild cognitive impairment improves diagnostic precision, biomarker associations, and progression rates. $J$ Alzheimers Dis 42, 275-289.

[4] Edmonds EC, Delano-Wood L, Clark L R, Jak AJ, Nation DA, McDonald CR, Libon DJ, Au R, Galasko D, Salmon DP, Bondi MW for the Alzheimer's Disease Neuroimaging Initiative (2015) Susceptibility of the conventional criteria for mild cognitive impairment to false-positive diagnostic errors. Alzheimers Dement 11, 415-424.

[5] Jak AJ, Bondi MW, Delano-Wood L, Wierenga C, CoreyBloom J, Salmon DP, Delis DC (2009) Quantification of five neuropsychological approaches to defining mild cognitive impairment. Am J Geriatr Psychiatry 17, 368-375.

[6] Binder LM, Iverson GL, Brooks BL (2009) To err is human: "Abnormal" neuropsychological scores and variability are common in healthy adults. Arch Clin Neuropsychol 24, 3146.

[7] Heaton RK, Miller SW, Taylor MJ, Grant I (2001) Revised Comprehensive Norms for an Expanded Halsted-Reitan battery: Demographically Adjusted Neuropsychological Norms for African American and Caucasian Adults. Psychological Assessment Resources, Odessa, FL.

[8] Taylor MJ, Heaton RK (2001) Sensitivity and specificity of WAIS-III/WMS-III demographically corrected factor scores in neuropsychological assessment. J Int Neuropsychol Soc 7, 867-874.

[9] Eckerström M, Göthlin M, Rolstad S, Hessen E, Eckerström C, Nordlund A, Johansson B, Svensson J, Jonsson M, Sacuiu S, Wallin A (2017) Longitudinal evaluation of criteria for subjective cognitive decline and preclinical Alzheimer's disease in a memory clinic sample. Alzheimers Dement (Amst) 8, 96-107.

[10] Vos SJB, Xiong C, Visser PJ, Jasielec MS, Hassenstab J, Grant EA, Cairns NJ, Morris JC, Holtzman DM, Fagan AM (2013) Preclinical Alzheimer's disease and its outcome: A longitudinal cohort study. Lancet Neurol 12, 957-965.

[11] Hessen E, Eckerström M, Nordlund A, Selseth Almdahl I, Stålhammar J, Bjerke M, Eckerström C, Göthlin M, Fladby T, Reinvang I, Wallin A (2017) Subjective cognitive impairment is a predominantly benign condition in memory clinic patients followed for 6 years: The GothenburgOslo MCI Study. Dement Geriatr Cogn Disord Extra 7, 1-14

[12] van Harten AC, Smits LL, Teunissen CE, Visser PJ, Koene T, Blankenstein MA, Scheltens P, van der Flier WM (2013) Preclinical AD predicts decline in memory and executive 
functions in subjective complaints. Neurology 81, 14091416.

[13] Sierra-Rio A, Balasa M, Olives J, Antonell A, Iranzo A, Castellví M, Bosch B, Grau-Rivera O, Fernandez-Villullas G, Rami L, Lladó A, Sánchez-Valle R, Molinuevo JL (2016) Cerebrospinal fluid biomarkers predict clinical evolution in patients with subjective cognitive decline and mild cognitive impairment. Neurodegener Dis 16, 69-76.

[14] Petersen RC, Caracciolo B, Brayne C, Gauthier S, Jelic V, Fratiglioni L (2014) Mild cognitive impairment: A concept in evolution. J Intern Med 275, 214-228.

[15] Hessen E, Reinvang I, Eliassen CF, Nordlund A, Gjerstad L, Fladby T, Wallin A (2014) The combination of dysexecutive and amnestic deficits strongly predicts conversion to dementia in young mild cognitive impairment patients: A report from the Gothenburg-Oslo MCI Study. Dement Geriatr Cogn Dis Extra 4, 76-85.

[16] Göthlin M, Eckerström M, Rolstad S, Wallin A, Nordlund A (2017) Prognostic accuracy of mild cognitive impairment subtypes at different cut-off levels. Dement Geriatr Cogn Disord 43, 330-341.

[17] Fladby T, Pålhaugen L, Selnes P, Waterloo K, Bråthen G, Hessen E, Almdahl IS, Arntzen KA, Auning E, Eliassen CF, Espenes R, Grambaite R, Grøntvedt GR, Johansen KK, Johnsen SH, Kalheim LF, Kirsebom BE, Müller KI, Nakling AE, Rongve A, Sando SB, Siafarikas N, Stav AL, Tecelao S, Timon S, Bekkelund SI, Aarsland D (2017) Detecting at-risk Alzheimer's disease cases. J Alzheimers Dis 60 , 97-105.

[18] Jessen F, Amariglio RE, van Boxtel M, Breteler M, Ceccaldi M, Chételat G, Dubois B, Dufouil C, Ellis KA, van der Flier WM, Glodzik L, van Harten AC, de Leon MJ, McHugh P, Mielke MM, Molinuevo JL, Mosconi L, Osorio RS, Perrotin A, Petersen RC, Rabin LA, Rami L, Reisberg B, Rentz DM, Sachdev PS, de la Sayette V, Saykin AJ, Scheltens P, Shulman MB, Slavin MJ, Sperling RA, Stewart R, Uspenskaya O, Vellas B, Visser PJ, Wagner M (2014) Subjective Cognitive Decline Initiative (SCD-I) Working Group: A conceptual framework for research on subjective cognitive decline in preclinical Alzheimer's disease. Alzheimers Dement 10, 844-852.

[19] Folstein MF, Folstein SE, McHugh PR (1975) "Mini-mental state". A practical method for grading the cognitive state of patients for the clinician. J Psychiatr Res 12, 189-198.

[20] Fillenbaum GG, van Belle G, Morris JC, Mohs RC, Mirra SS, Davis PC, Tariot PN, Silverman JM, Clark CM, WelshBohmer KA, Heyman A (2008) CERAD (Consortium to Establish a Registry for Alzheimer's Disease) The first 20 years. Alzheimers Dement 4, 96-109.

[21] Sotaniemi M, Pulliainen V, Hokkanen L, Pirttila T, Hallikainen I, Soininen H, Hanninen T (2012) CERADneuropsychological battery in screening mild Alzheimer's disease. Acta Neurol Scand 125, 16-23.

[22] Warrington EK, James M (1991) The Visual Object and Space Perception Battery. Thames Valley Test Company, Bury St Edmunds, England.

[23] Reitan RM, Wolfson D (1985) The Halstead-Reitan Neuropsychological Test Battery. Neuropsychology Press, Tucson.

[24] Benton AL, Hamsher K (1989) Multilingual Aphasia Examination. AJA Associates, Iowa City.

[25] Sjogren M, Vanderstichele H, Agren H, Zachrisson, O, Edsbagge, M, Wikkelso, C, Skoog, I, Wallin, A, Wahlund, LO Marcusson, J, Nägga, K (2001) Tau and Abeta42 in cerebrospinal fluid from healthy adults 21-93 years of age:
Establishment of reference values. Clin Chem 47, 17761781.

[26] Kalheim LF, Fladby T, Coello C, Bjornerud A, Selnes P (2018) [18F]-flutemetamol uptake in cortex and white matter: Comparison with cerebrospinal fluid biomarkers and [18F]-fludeoxyglucose. J Alzheimers Dis 62, 1595-1607.

[27] Alexopoulos, P, Kriett, L, Haller, B, Klupp, E, Gray, K, Grimmer, T, Laskaris N, Förster S, Perneczky R, Kurz A, Drzezga A, Fellgiebel A, Yakushev I; Alzheimer's Disease Neuroimaging Initiative (2014) Limited agreement between biomarkers of neuronal injury at different stages of Alzheimer's disease. Alzheimers Dement 10, 684-689.

[28] Vos SJ, Verhey F, Frolich L, Kornhuber J, Wiltfang J, Maier W, Peters O, Rüther E, Nobili F, Morbelli S, Frisoni GB, Drzezga A, Didic M, van Berckel BN, Simmons A, Soininen H, Kloszewska I, Mecocci P, Tsolaki M, Vellas B, Lovestone S, Muscio C, Herukka SK, Salmon E, Bastin C, Wallin A, Nordlund A, de Mendonça A, Silva D, Santana I, Lemos R, Engelborghs S, Van der Mussele S; Alzheimer's Disease Neuroimaging Initiative, Freund-Levi Y, Wallin ÅK, Hampel H, van der Flier W, Scheltens P, Visser PJ (2015) Prevalence and prognosis of Alzheimer's disease at the mild cognitive impairment stage. Brain 138, 1327-1338.

[29] Chetelat G, La Joie R, Villain N, Perrotin A, de La Sayette V, Eustache F, Vandenberghe R (2013) Amyloid imaging in cognitively normal individuals, at-risk populations and preclinical Alzheimer's disease. Neuroimage Clin 2, 356365.

[30] Kern S, Zetterberg H, Kern, J, Zettergren A, Waern M, Höglund K, Andreasson U, Wetterberg H, BörjessonHanson A, Blennow K, Skoog I (2018) Prevalence of preclinical Alzheimer disease. Neurology 90, e1682-e1691.

[31] Edmonds EC, Eppig J, Bondi MW, Leyden KM, Goodwin B, Delano-Wood L, McDonald CR (2016) Heterogeneous cortical atrophy patterns in MCI not captured by conventional diagnostic criteria. Neurology $\mathbf{8 7}$, 2108-2116.

[32] Jedynak BM, Lang A, Liu B, Katz E, Zhang Y, Wyman BT, Raunig D, Jedynak CP, Caffo B, Prince JL; Alzheimer's Disease Neuroimaging Initiative (2012) A computational neurodegenerative disease progression score: Method and results with the Alzheimer's Disease Neuroimaging Initiative Cohort. Neuroimage 63, 1478-1486.

[33] Ewers M, Walsh C, Trojanowski JQ, Shaw LM, Petersen RC, Jack CR Jr, Feldman HH, Bokde AL, Alexander GE, Scheltens P, Vellas B, Dubois B, Weiner M, Hampel H; North American Alzheimer's Disease Neuroimaging Initiative (ADNI) (2012) Prediction of conversion from mild cognitive impairment to Alzheimer's disease dementia based upon biomarkers and neuropsychological test performance. Neurobiol Aging 33, 1203-1214.

[34] Li K, Chan W, Doody RS, Quinn J, Luo S, Alzheimer's Disease Neuroimaging Initiative (2017) Prediction of conversion to Alzheimer's disease with longitudinal measures and time-to-event data. $J$ Alzheimers Dis 58, 361-371.

[35] Moradi E, Hallikainen I, Hanninen T, Tohka J, Alzheimer's Disease Neuroimaging Initiative (2017) Rey's Auditory Verbal Learning Test scores can be predicted from whole brain MRI in Alzheimer's disease. Neuroimage Clin 13, 415-427.

[36] Eckerstrom C, Olsson E, Klasson N, Berge J, Nordlund A, Bjerke M, Wallin A (2015) Multimodal prediction of 
dementia with up to 10 years follow up: The Gothenburg MCI study. J Alzheimers Dis 44, 205-214.

[37] Jack CR Jr, Bennett DA, Blennow K, Carrillo MC, Dunn B, Haeberlein SB, Holtzman DM, Jagust W, Jessen F, Karlawish J, Liu E, Molinuevo JL, Montine T, Phelps C, Rankin KP,
Rowe CC, Scheltens P, Siemers E, Snyder HM, Sperling R (2018) NIA-AA Research Framework: Toward a biological definition of Alzheimer's disease. Alzheimers Dement 14, 535-562. 